\title{
CARACTERIZACIÓN Y CLASIFICACIÓN BOTÁNICA DE VEINTIDOS LÍNEAS DE MANÍ (Arachis hypogaea L.) EVALUADAS EN LA PROVINCIA DE NUBLE, CHILE
}

\section{CHARACTERIZATION AND BOTANICAL CLASSIFICATION OF TWENTY- TWO PEANUT LINES (Arachis hypogaea L.) EVALUATED IN ÑUBLE PROVINCE, CHILE}

\author{
Nelson Zapata ${ }^{1 *}$, Luis Henriquez ${ }^{1}$, y Víctor L. Finot ${ }^{1}$ \\ ${ }^{1}$ Facultad de Agronomía, Universidad de Concepción, Casilla 537, Chillán, Chile. \\ *Autor para correspondencia: E-mail: nzapata@udec.cl.
}

\section{RESUMEN}

En Chile no existen cultivares de maní (Arachis hypogaea L.) mejorados, por ello es necesario evaluar genotipos para el desarrollo de variedades comerciales adaptadas a las condiciones climáticas locales. El objetivo de este estudio fue caracterizar y clasificar 22 líneas avanzadas de maní según variedad botánica y tipo comercial, y su adaptabilidad en la Provincia de Ñuble, Chile. Se evaluaron 23 descriptores morfológicos, 17 cuantitativos y 6 cualitativos, a los que se realizó análisis estadístico uni y multivariado. Los resultados obtenidos permitieron agrupar las líneas en la subsp. hypogaea var. hypogaea y la subsp. fastigiata var. fastigiata. La primera se divide en dos subgrupos: uno formado por las líneas 4, 15, 17, 18, 41 y 42 (tipo Runner) y otro formado por las líneas 3 y 8 (tipo Virginia). Por su parte, la subsp. fastigiata var. fastigiata está conformada por las líneas 1, 5, 7, 10, 11, 12, 13, 14, 16, 19, 20, 21, 22 y 23 (tipo Valencia). La subsp. hypogaea presentó crecimiento decumbente, ramificación alternada y frutos con dos semillas de color castaño. La subsp. fastigiata presentó crecimiento erecto, ramificación secuencial y frutos con tres semillas de color rojo. Las líneas 3, 18 y 20 mostraron mejor adaptabilidad a las condiciones climáticas de la Provincia de Ñuble.

Palabras clave: Arachis hypogaea, maní tipo Valencia, maní tipo Runner, maní tipo Virginia.

\section{ABSTRACT}

There are no improved peanut cultivars (Arachis hypogaea L.) in Chile. Therefore, genotypes need to be evaluated in order to develop commercial varieties adapted to local conditions. The objective of this study was to characterize and classify twenty-two advanced peanut lines according to their botanical and commercial variety, and adaptability in Nuble Province, Chile. Twentythree morphological descriptors (17 quantitative and 6 qualitative) were evaluated, and data were analyzed by uni- and multivariate analyses. The results allowed classifying the lines into subspecies hypogaea var. hypogaea and fastigiata var. fastigiata. The former was divided in two subgroups: one consisting of lines $4,15,17,18,41,42$ (type Runner), and the other consisting of lines 3 and 8 (type Virginia). Subsp. fastigiata var. fastigiata included lines 1, 5, 7, 10, 11, 12, 13, 14, 16, 19, 20, 21, 22, 23 (type Valencia). Subsp. hypogaea has decumbent growing, alternate branching and fruits with two brown seeds. Subsp. fastigiata has erect growing, sequential branching and fruits with three red seeds. Lines 3, 18 and 20 showed better adaptability to local conditions in Nuble Province, Chile.

Key words: Arachis hypogaea, peanut type Valencia, peanut type Runner, peanut type Virginia. 


\section{INTRODUCCIÓN}

Arachis hypogaea L. es una especie dicotiledónea que pertenece a la familia Fabaceae, subfamilia Papilionoideae. Es originaria de Sudamérica, específicamente entre el noroeste de Argentina $\mathrm{y}$ el sur de Bolivia (Ferguson et al., 2004; Krapovickas et al., 2009). Es una planta herbácea de crecimiento vegetativo erecto, decumbente o rastrero, que posee dos patrones de ramificación: a) alterna, con crecimiento vegetativo postrado o decumbente, y b) secuencial, con crecimiento arbustivo y compacto. La clasificación taxonómica está basada en la presencia o ausencia de flores y ramas laterales sobre el eje central de la planta (Krapovickas y Gregory, 1994).

El maní cultivado comprende seis variedades botánicas, reunidas en dos subespecies: subsp. hypogaea Waldron y subsp. fastigiata Waldron. La primera incluye las variedades hypogaea e hirsuta Köhler, en tanto la segunda incluye las variedades fastigiata, vulgaris C. Harz, peruviana Krapov. \& W. C. Gregory y aequatoriana Krapov. $\&$ W. C. Gregory. Por otra parte, a nivel comercial se distinguen cuatro tipos de maní: Runner y Virginia (var. hypogaea), Español (var. vulgaris) y Valencia (var. fastigiata) (Fernández y Giayetto, 2006). Las tres últimas son las más cultivadas en el mundo (Holbrook y Stalker, 2003).

El sistema radicular del maní está formado por una raíz principal que alcanza 30 a $60 \mathrm{~cm}$ de largo y raíces laterales (Fernández y Giayetto, 2006). El tallo puede ser angular o cilíndrico, con entrenudos marcados por la inserción de las ramas y hojas que pueden ser glabras o pubescentes según la variedad botánica. Las hojas son estipuladas y tetrafoliadas, en tanto las inflorescencias, que se originan en los nudos reproductivos, tienen de tres a cinco flores, generalmente con corola amarilla, que se encuentran sobre las ramificaciones o tallo principal, dependiendo de la variedad. La floración abarca el $80 \%$ del periodo vegetativo del cultivo traslapándose con la fructificación (Fernández y Giayetto, 2006). Una vez producida la fecundación se alarga la base del ovario, generando una estructura denominada ginóforo, que penetra en el suelo y lleva en su extremo el o los óvulos fecundados. Los frutos son vainas indehiscentes y lomentiformes, y debido a la floración indeterminada, al momento de la cosecha se encuentran en diferentes estados de desarrollo. Las semillas son alargadas o redondeadas, con tegumento membranáceo.

Se cultiva desde los $40^{\circ}$ de latitud norte y los $40^{\circ}$ de latitud sur (Fernández y Giayetto, 2006), es de establecimiento primaveral y no tolera heladas. La duración del ciclo vegetativo varía según el cultivar y las temperaturas ambientales; existen cultivares de ciclos vegetativos largos (170 a 180 días), intermedios (120 a 140 días) y también de ciclos vegetativos cortos (80 a 120 días) (SánchezDomínguez et al., 2006; Zapata et al., 2012).

La precocidad es un aspecto de gran importancia ya que se relaciona estrechamente con el rendimiento (Fernández y Giayetto, 2006). Un indicador de precocidad es la proporción de frutos maduros por planta que hayan alcanzado la madurez fisiológica en un determinado período de tiempo; otro indicador es el número de días después de siembra, el cual se relaciona con la acumulación de grados día (tiempo térmico). Adelantar la fecha de cosecha es más perjudicial que su retraso, traduciéndose en disminución del rendimiento (15 a $21 \%$ ) y del tamaño de grano cosechado (5 a 6\%) (Fernández y Giayetto, 2006).

En Chile no existen cultivares comerciales de maní, lo que limita en gran medida el desarrollo del cultivo en el país (Zapata et al., 2012). Las semillas que utilizan los agricultores para su siembra provienen de la selección que hacen de su propia cosecha año tras año, al cual se le denomina maní chileno. El maní chileno se caracteriza por poseer un hábito de crecimiento arbustivo y un largo periodo vegetativo (160 a 180 días, según la localidad) con tres a seis semillas por vaina y que puede corresponder a una mezcla de maní tipo Español y Valencia.

Por ello, y considerando que este cultivo es una alternativa productiva para los agricultores, es necesario evaluar genotipos para ser usados en el desarrollo de variedades comerciales de maní chileno de alta productividad.

La presente investigación tuvo como objetivo caracterizar y clasificar 22 líneas avanzadas de maní según variedad botánica y comercial, y además evaluar su adaptación a las condiciones climáticas de la Provincia de Nuble.

\section{MATERIALES Y MÉTODOS}

\section{Genotipos}

Los genotipos a evaluar corresponden a 22 líneas avanzadas de maní seleccionadas en forma masal por nueve generaciones, en la Facultad de Agronomía de la Universidad de Concepción durante ocho años, las cuales provenían de 40 accesiones originales del extinto programa de Oleaginosas de Grano de INIA, La Platina, Santiago de Chile.

\section{Ubicación y características edafoclimáticas del sitio experimental}

El establecimiento de las líneas se realizó en la Estación Experimental El Nogal, Chillán (36ํㅜㄴ'S; $72^{\circ} 06^{\prime} \mathrm{O}, 144$ m.s.n.m.). El clima es mediterráneo, con precipitación anual de 1.000 a $1.300 \mathrm{~mm}$, 
temperatura media anual de $14^{\circ} \mathrm{C}$ y periodo libre de heladas de cinco a seis meses (Del Pozo y Del Canto, 1999). El suelo pertenece a la serie Arrayán (Medial, thermic Humic Melanoxerand), con topografía plana a suavemente ondulada y buen drenaje (Stolpe, 2006).

\section{Establecimiento y manejo agronómico}

Cada línea se estableció empleando un diseño de bloques completos al azar, con tres repeticiones. Cada parcela comprendió tres hileras de 3 metros de largo y espaciadas $60 \mathrm{~cm}\left(5,4 \mathrm{~m}^{2}\right)$. El suelo se preparó secuencialmente con arado vertedera y dos rastrajes con rastra de discos. Antes de la siembra, la semilla de cada accesión fue desinfectada con fungicida Fludioxonilo 2,5\% i.a. y Mefenoxam 1,0\% i.a. (Celest ${ }^{\circledR}$ XL 035 FS) e insecticida Fipronil $25 \%$ i.a. (Donau ${ }^{\circledR} 25$ SC). La siembra se realizó la primera semana de noviembre. Empleando un binador se abrieron surcos de hasta $8 \mathrm{~cm}$ de profundidad. Considerando análisis químico de suelo previo (pH: 6,8; M.O.: 6,2 \%; $\mathrm{N}$ disponible: $8,6 \mathrm{mg} \mathrm{kg}$; P Olsen: $12,5 \mathrm{mg} \mathrm{kg}^{-1}$; $\mathrm{K}$ disponible: $\left.145 \mathrm{mg} \mathrm{kg}^{-1}\right)$, se suministró una fertilización base en forma localizada en el surco de siembra, consistente en $250 \mathrm{~kg} \mathrm{ha}^{-1}$ de Mezcla 9-41-12 y $130 \mathrm{~kg} \mathrm{ha}^{-1}$ de urea. Una vez incorporados los fertilizantes se distribuyeron las semillas no inoculadas con bacterias fijadoras de nitrógeno a una distancia de $8 \mathrm{~cm}$ sobre la hilera. En las etapas de prefloración (diciembre) e inicios de floración (enero) se aplicó nuevamente al cultivo $130 \mathrm{~kg} \mathrm{ha}^{-1}$ de urea. El cultivo se mantuvo libre de malezas mediante control manual. A partir de diciembre $y$ hasta febrero se efectuaron riegos por surco con una frecuencia semanal. Además, se realizaron dos aporcas a inicios de floración y transcurridos 20 días de realizada la primera. Cada genotipo fue cosechado transcurridos 150 días desde siembra (dds).

\section{Caracterización morfológica}

Para la caracterización morfológica, 112 días después de siembra, desde la hilera central de cada parcela se extrajo una planta (Tabla 1). Para los caracteres vegetativos: hábito de crecimiento, patrón de ramificación, altura de la planta, ramas primarias por planta y número de nudos del tallo principal se evaluó una muestra por repetición. Para los caracteres morfológicos: forma del folíolo, pilosidad del folíolo, pilosidad del tallo, largo del pecíolo, largo del folíolo, y ancho del folíolo, se consideraron diez muestras por repetición.

A cosecha (150 dds) se muestrearon frutos completamente maduros y secos. Para los caracteres reproductivos se evaluaron 10 muestras por repetición, excepto para el parámetro peso de 100 frutos, para el cual se consideraron tres muestras. Ambas evaluaciones fueron tratadas como datos cualitativos de tipo nominal y además se determinaron datos cuantitativos de tipo continuo (Tabla I) (Franco e Hidalgo, 2003). Cada observación fue descrita según pauta del Consejo Internacional de Recursos Fitogenéticos (IBPGR, 1985).

\section{Porcentaje de frutos maduros e índice de cosecha}

Al momento de la cosecha se extrajo una planta por parcela a la cual se le determinó el número y peso seco total de frutos, número y peso seco de frutos maduros, según Fernández y Giayetto (2006) y peso seco total del residuo no cosechable. Con los datos obtenidos se calculó el porcentaje de frutos maduros e índice de cosecha según Satorre et al. (2003).

También se cuantificó el tiempo térmico acumulado para todo el ciclo de cultivo expresado como días grados acumulados (DGA). Para ello se utilizaron los datos de temperatura registrados por la Estación Meteorológica de la Facultad de Ingeniería Agrícola de la Universidad de Concepción, ubicada en el Campus Chillán, distante a $400 \mathrm{~m}$ del sitio experimental. Los DGA fueron determinados mediante la fórmula

DGA $=[($ T. máx. + T. min. $) / 2]-\mathrm{T}$. umbral

donde:

T. máx.= Temperatura máxima diaria $\left({ }^{\circ} \mathrm{C}\right)$;

T. mín. $=$ Temperatura mínima diaria $\left({ }^{\circ} \mathrm{C}\right)$;

T. umbral $=$ Temperatura umbral inferior $\left({ }^{\circ} \mathrm{C}\right)$. Como T. umbral se consideró $10^{\circ} \mathrm{C}$ (Fernández y Giayetto, 2006).

\section{Análisis de datos}

El análisis de los datos se realizó mediante la aplicación de métodos multivariados y univariados. En el análisis de componentes principales, las variables cuantitativas fueron estandarizadas por filas y columnas. Las variables cuantitativas y cualitativas fueron sometidas a análisis de conglomerados y discriminante con el objeto de obtener grupos de clasificación. El análisis de conglomerados se llevó a cabo con el algoritmo de la media aritmética no ponderada (UPGMA, unweighted pair-group method using arithmetic averages), utilizando el coeficiente de distancia de Gower. Los datos cuantitativos continuos fueron sometidos a análisis de varianza. Las medias fueron comparadas mediante el test de Duncan con un nivel de confianza del 95\%. Para todos los análisis se utilizó el software Infostat (Di Rienzo et al., 2013).

\section{RESULTADOS Y DISCUSIÓN}

\section{Análisis de componentes principales}

Los dos primeros componentes explican 
Tabla 1. Caracteres morfológicos utilizados en la caracterización de 22 líneas de maní en la Provincia de Nuble, Chile.

Table 1. Morphological characters used to characterize 22 peanut lines in the Nuble Province, Chile.

\begin{tabular}{l} 
Caracteres Descripción ${ }^{1}$; Abreviatura \\
Vegetativos \\
1.- Hábito crecimiento: Decumbente (1), Erecto (2), Rastrero (3); H Cr \\
2.- Patrón ramificación: Alternado (1), Secuencial (2); P Ra \\
3.- Forma folíolos: Elíptico (1); For Fo \\
4.- Pilosidad folíolos: Piloso (1); Pi Fo \\
5.- Pilosidad del tallo: Piloso (1); Pi T \\
6.- Altura de la planta: Centímetros (cm); A Pl \\
7.- Ramas primarias por planta: Número; N ${ }^{\circ}$ Ra $1^{\circ}$ \\
8.- Nudos del tallo principal: Número; $\mathrm{N}^{\circ} \mathrm{NuTP}$ \\
9.- Largo del pecíolo: Centímetros (cm); L Pe \\
10.- Largo del folíolo: Centímetros (cm); L Fo \\
11.- Ancho del folíolo: Centímetros (cm); A Fo \\
\hline Reproductivos: Frutos y semillas \\
12.- Color primario de la semilla: Castaño claro (1), Rojo oscuro (2); Co S \\
13.- Largo frutos: Centímetros (cm); LF \\
14.- Ancho frutos: Centímetros (cm); AF \\
15.- Largo semillas: Centímetros (cm); LS \\
16.- Ancho semillas: Centímetros (cm); AS \\
17.- Peso de 100 frutos: Gramos (g); P100F \\
18.- Peso de 100 semillas: Gramos (g); P100S \\
19.- Semillas por fruto: Número; N ${ }^{\circ}$ SXF \\
20.- Relación semilla/fruto base peso: Porcentaje (\%); \% S/F P \\
\hline Reproductivos: Porcentaje de frutos maduros e índice de cosecha \\
21.- Fruto maduro base número: Porcentaje (\%); \% FMBN ${ }^{\circ}$ \\
22.- Fruto maduro base peso: Porcentaje (\%); \% FMBP \\
23.- Índice de cosecha: Porcentaje (\%); \% IC \\
\hline${ }^{1}$ IBPGR, 1985.
\end{tabular}

el $32,3 \%$ y el $22,0 \%$ de la varianza total, respectivamente, lo que acumulado corresponde al $54,3 \%$ (Tabla 2). Las variables que más aportaron al primer componente fueron largo de frutos $(0,38)$, relación semilla/fruto base peso $(-0,36)$, peso de 100 frutos $(0,32), \mathrm{N}^{\circ}$ semillas por fruto $(0,32)$, ancho de frutos $(0,30)$, porcentaje de fruto maduro base peso $(0,29)$ y altura de la planta $(0,26)$. Las variables que más aportaron al segundo componente son el largo de las semillas $(0,41)$, peso de 100 semillas $(0,41)$, ancho de las semillas $(0,35)$, altura de la planta $(-0,30)$, ancho de frutos $(0,30)$ e índice de cosecha $(0,29)$. La distribución de las 22 líneas de maní y las 17 variables cuantitativas en el plano formado por los dos primeros componentes se muestra en la Fig. 1, en la que se observan tres grupos. El primer componente (CP1) separa las líneas 1, 5, $7,10,11,12,13,14,16,19,20,21,22$ y 23 hacia el extremo positivo del eje; éstas se caracterizan por tener frutos largos y anchos, mayor número de semillas por fruto, alto peso de 100 frutos, alta proporción de frutos maduros base número a los 150 días después de siembra y plantas de mayor altura. Por su parte, las líneas 4, 15, 17, 18, 41 y 42 se separan hacia el extremo negativo del primer componente; éstas se caracterizan por poseer alta relación semilla/fruto. El segundo componente $(\mathrm{CP} 2)$ separa las líneas 3 y 8 ; que presentan semillas más grandes (largo y ancho) y altos valores de peso de 100 semillas e índice de cosecha.

Para el análisis de conglomerados (AC) se utilizaron las variables cuantitativas que más contribuyeron a los dos primeros componentes: largo de semillas, peso de 100 semillas, largo de frutos, relación semilla/fruto base peso, ancho de semillas, peso de 100 frutos y $\mathrm{N}^{\circ}$ semillas por fruto, y las variables cualitativas: hábito de crecimiento, patrón de ramificación y color de semilla. El AC separa las 22 líneas de maní en dos grupos (Fig. 2). El primer grupo (A), está 
Tabla 2. Contribución (autovectores) de variables cuantitativas a los dos primeros componentes principales (22 líneas, 17 variables).

Table 2. Contributions (eigenvectors) of quantitative variables to the first principal components (22 lines, 17 variables).

\begin{tabular}{|c|c|c|c|c|c|}
\hline Componente Principal & 1 & 2 & Componente Principal & 1 & 2 \\
\hline \multicolumn{3}{|l|}{ Caracterización vegetativa } & \multicolumn{3}{|c|}{ Caracterización frutos y semillas } \\
\hline Altura planta & 0,26 & $-0,30$ & Largo semillas & 0,13 & 0,41 \\
\hline № ramas primarias & $-0,11$ & $-0,12$ & Ancho semillas & $-0,11$ & 0,35 \\
\hline № nudos tallo principal & 0,27 & $-0,25$ & Peso 100 frutos & 0,32 & 0,24 \\
\hline Largo peciolo & 0,11 & $-0,02$ & Peso 100 semillas & $-0,13$ & 0,41 \\
\hline Largo folíolo & 0,23 & 0,21 & № semillas por fruto & 0,32 & $-0,23$ \\
\hline Ancho folíolo & 0,15 & 0,20 & $\% \mathrm{~S} / \mathrm{F} \mathrm{P}$ & $-0,36$ & 0,03 \\
\hline \multicolumn{3}{|c|}{ Caracterización frutos y semillas } & \multicolumn{3}{|c|}{ Frutos maduros e índice de cosecha } \\
\hline Largo frutos & 0,38 & 0,07 & \% FMBNo & 0,25 & $-0,08$ \\
\hline \multirow[t]{2}{*}{ Ancho frutos } & 0,30 & 0,30 & $\%$ FMBP & 0,29 & $-0,01$ \\
\hline & & & $\%$ IC & 0,04 & 0,29 \\
\hline
\end{tabular}

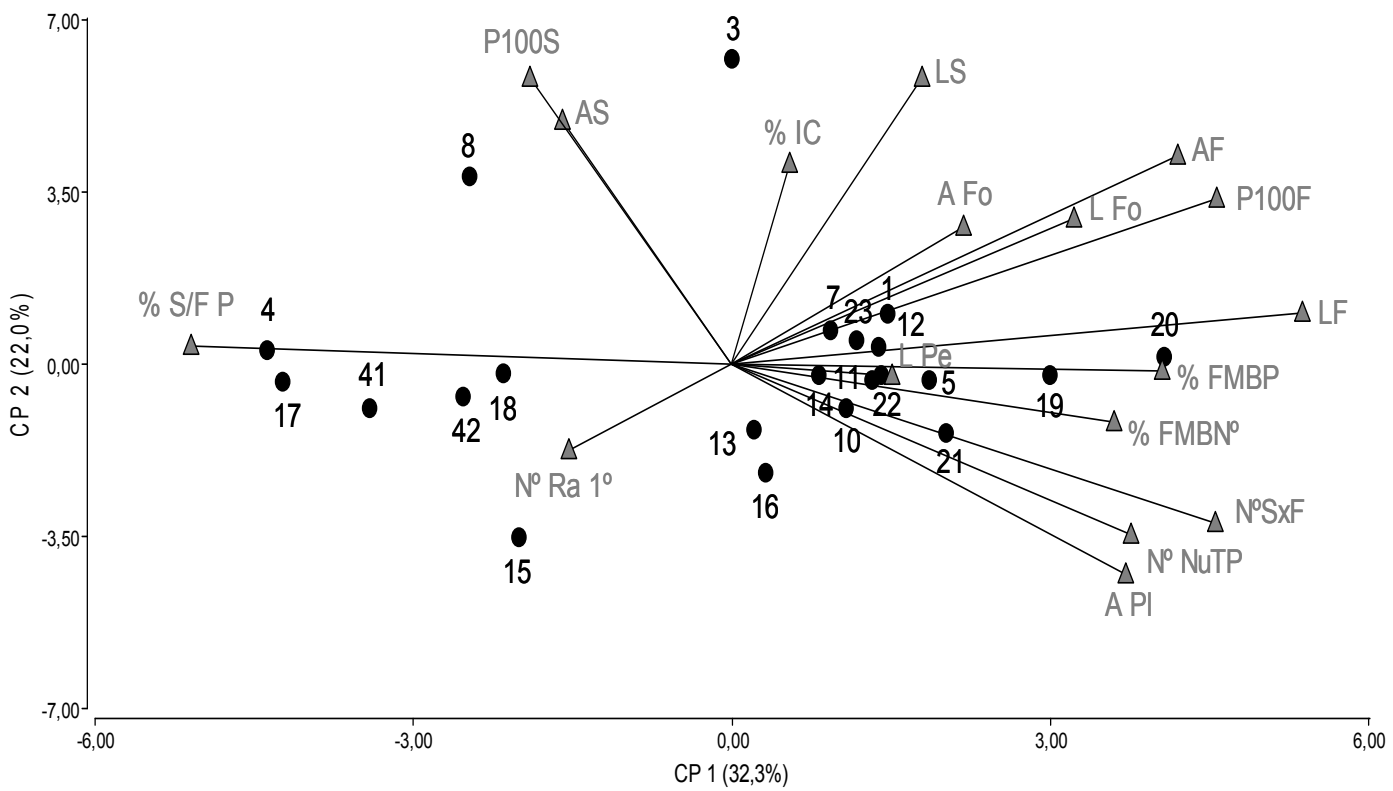

Fig. 1. Biplot mostrando distribución de 22 líneas de maní (círculo negro) y 17 variables cuantitativas (triángulo gris) en el plano formando por los dos primeros componentes principales (correlación cofenética $\mathbf{0 , 9 2 ) .}$

Fig. 1. Biplot showing distribution of 22 peanut lines (black circle) and 17 quantitative variables (gray triangle) in the plane formed by the first principal component (cophenetic correlation 0.92).

formado por ocho líneas y se puede dividir en los subgrupos: A1, formado por las líneas 4, 15, 17, 18, 41 y 42; y A2 compuesto por las líneas 3 y 8 . El segundo grupo (B) está formado por las líneas 1 , 5, 7, 10, 11, 12, 13, 14, 16, 19, 20, 21, 22 y 23.

Por sus características botánicas, el grupo A corresponde a la subespecie hypogaea var. hypogaea. Estas líneas se caracterizaron por presentar hábito de crecimiento rastrero o decumbente, ramificación alternada y presentar dos semillas por fruto (Krapovickas y Gregory, 1994; Harch et al. 1999). La diferencia que se observa entre los subgrupos A1 y A2 se debe a las mayores dimensiones que presenta la semilla (largo, ancho) y peso de 100 semillas de las líneas del grupo A2 $(3,8)$, característica 


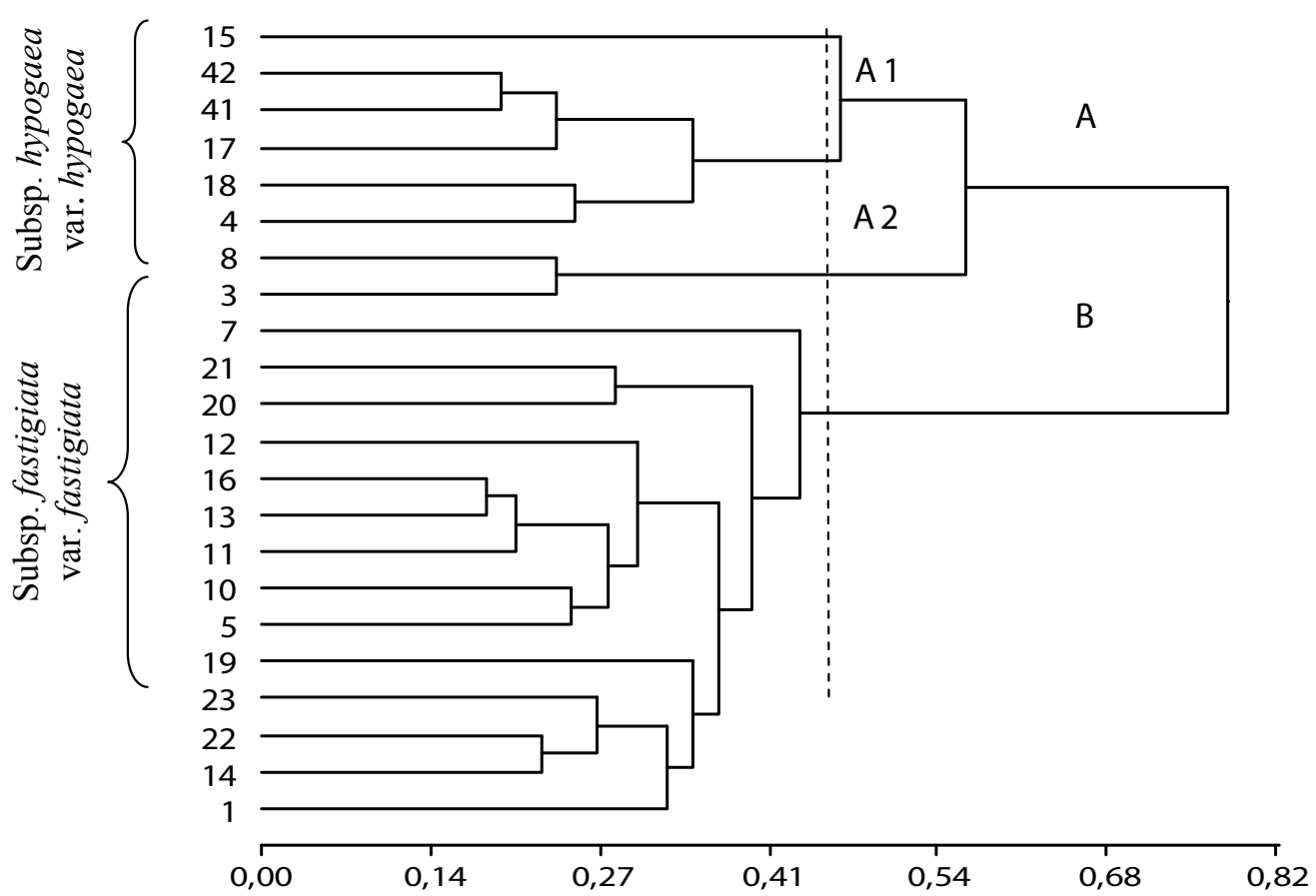

Fig 2. Dendrograma de 22 líneas de maní utilizando siete descriptores cuantitativos y tres descriptores cualitativos (correlación cofenética: 0,92 ).

Fig 2. Dendrogram of 22 peanut lines using seven quantitative and three qualitative descriptors (cophenetic correlation 0.92).

que corresponde al maní tipo Virginia (Soave et al., 2013). Las líneas del grupo B, subespecie fastigiata var. fastigiata, tipo comercial Valencia, se caracterizaron por poseer hábito de crecimiento erecto, ramificación secuencial y tres semillas por fruto, características que concuerdan con las descritas para esta variedad por Harch et al. (1999) y Ferguson et al. (2004).

\section{Caracterización vegetativa}

La altura de planta varió entre 12,7 y $33,3 \mathrm{~cm}$ (Tabla 3). Las líneas 1, 5, 7, 10, 11, 12, 13, 14, 16, 19, 20, 21, 22 y 23, alcanzaron mayor altura que el resto de las líneas. Esta diferencia se explicaría por el hábito de crecimiento que presentan las distintas líneas; en general, las plantas con hábito de crecimiento rastrero o decumbente presentan menores alturas que aquellas con hábito de crecimiento erecto. Los resultados obtenidos en el presente estudio son similares a los encontrados por Upadhyaya et al. (2006) y Zapata et al. (2012) con distintas accesiones estudiadas. El número de ramas primarias varió de 4 a 6; el número de nudos del tallo principal varió de 9 a 14, el largo del peciolo varió de 5,1 a 7,5 cm, el largo y ancho del folíolo apical variaron entre 3,7 a $5,5 \mathrm{~cm}$ y de 1,77 a $2,70 \mathrm{~cm}$, respectivamente.

En cuanto al hábito de crecimiento, las líneas 1,5, $7,10,11,12,13,14,16,19,20,21,22$ y 23 presentaron crecimiento erecto, característico de la subsp. fastigiata var. fastigiata maní tipo Valencia, la línea 17 presentó hábito de crecimiento rastrero, y el resto de las líneas presentaron crecimiento decumbente, característico de la subsp. hypogaea var. hypogaea manítipos Virginia y Runner (Fernández y Giayetto, 2006). En relación al patrón de ramificación, éste fue secuencial en las líneas 1, 5, 7, 10, 11, 12, 13, 14, 16, 19, 20, 21, 22 y 23, en cambio el resto de las líneas presentaron ramificación alternada (Tabla 3). Estas características son concordantes con lo descrito por Fernández y Giayetto (2006) para diversos tipos de maní. Cabe señalar que todas las líneas de maní estudiadas presentaron folíolos elípticos pilosos y tallo piloso.

\section{Caracterización reproductiva \\ Frutos y semillas}

Los caracteres peso de 100 frutos, largo y ancho de frutos presentaron en las líneas 4, 15, 17, 18, 41 y 42, los menores valores, los cuales fluctuaron entre 104,9 y $121,8 \mathrm{~g} ; 2,90$ y $3,17 \mathrm{~cm} ; 1,23$ y $1,40 \mathrm{~cm}$, respectivamente (Tabla 4). El resto de las líneas de maní presentaron frutos con rangos de peso y de longitud superiores a las líneas de maní anteriormente mencionadas. Con respecto al peso de 100 frutos sobresalen las líneas 3, 8 y 20 con pesos de 159,1; 150,1 y 174,7 g, respectivamente.

En relación al carácter peso de 100 semillas, las líneas 3 y 8 presentaron un peso significativamente 


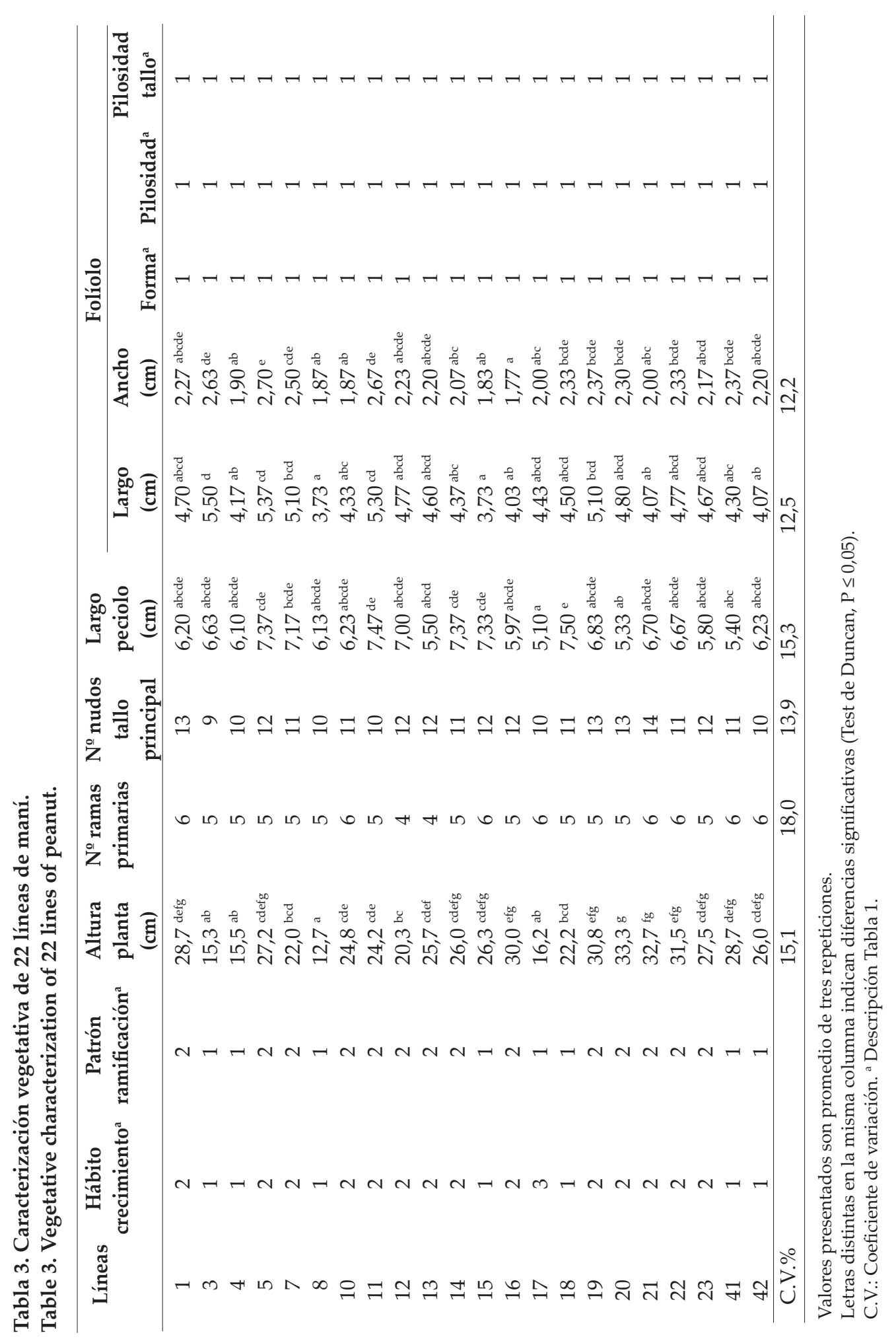




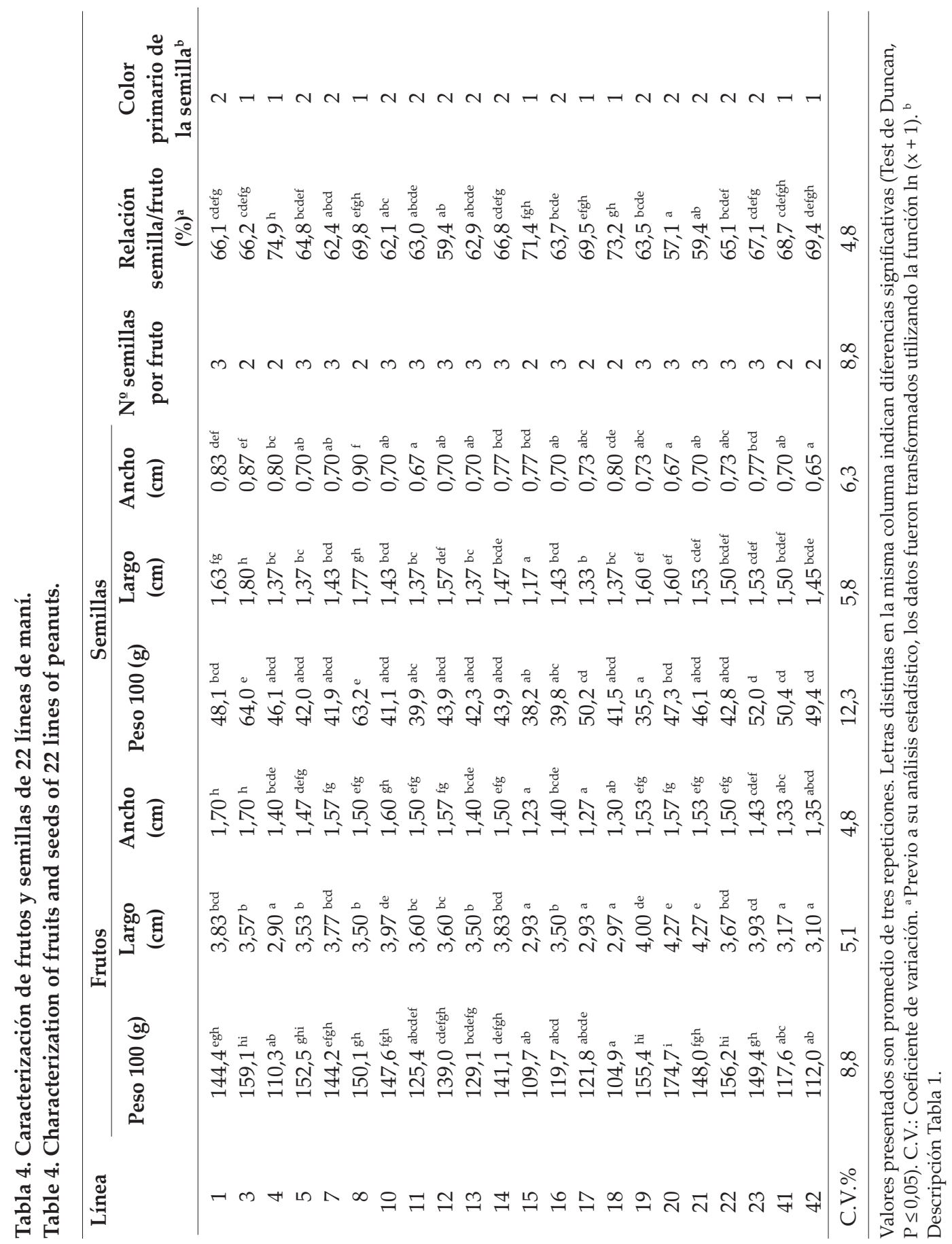

mayor al resto de las líneas, variando entre 63,2 y $64,0 \mathrm{~g}$, mientras que el resto de las líneas presentó pesos que fluctuaron entre 35,5 y $50,4 \mathrm{~g}$.

El peso de 100 frutos y el peso de 100 semillas obtenidos en el presente estudio resultaron más acotados que los informados por Pascual et al.
(2006), quienes obtuvieron valores entre 46,8 y 120,2 g en 10 accesiones de maní de la colección de germoplasma de Perú. A su vez, SánchezDomínguez et al. (2006) obtuvieron valores entre 26,7 y 83,2 g en 64 accesiones en México, lo cual da cuenta de la gran variabilidad que es posible 
encontrar respecto al peso de las semillas en esta especie.

En relación al largo y ancho de semillas, los valores obtenidos en las líneas 3 y 8 fueron significativamente mayores al resto de las líneas, alcanzando valores de 1,77 a 1,80 cm de largo y de 0,87 a 0,90 $\mathrm{cm}$ de ancho, respectivamente. En el resto de las líneas de maní el ancho de semilla varió de 1,17 a 1,80 $\mathrm{cm}$ y el largo de 0,67 a $0,89 \mathrm{~cm}$. Estos valores están dentro de los rangos de variación obtenidos por Mazzani et al. (2009) y Zapata et al. (2012), quienes informaron variaciones entre 0,9 a 4,2 en el largo y entre 0,6 hasta $1,8 \mathrm{~cm}$ en el ancho, de semillas de maní con datos obtenidos de 546 y 20 accesiones estudiadas en Venezuela y Chile, respectivamente.

El número de semillas por fruto varió entre 2 y 3 para las diferentes líneas evaluadas. (Tabla 4). Las líneas 3, 4, 8, 15, 17, 18, 41 y 42 presentaron dos semillas por fruto, mientras que el resto de las líneas presentaron tres semillas por fruto. Por su parte, Upadhyaya et al. (2002), Méndez-
Natera y Osorio (2003), Upadhyaya et al. (2006) y Zapata et al. (2012) encontraron de 1 a 3 semillas por fruto en las distintas accesiones de maní estudiadas. La relación semilla/fruto fluctuó entre 57,1 y 74,9\%, siendo las líneas 4, 15, 17, 18, 41, 42 las que mostraron mayores valores, presentando rangos entre 68,7 y $74,9 \%$. Estos resultados son levemente superiores a los informados por Escobar y Bustamante (1997), que encontraron rangos entre 48 a $61 \%$ de semilla/fruto en 11 genotipos de maní estudiados en Colombia, mientras que Zapata et al. (2012) encontraron valores entre 64 a 73\%.

Para el parámetro color de la semilla, las líneas de maní 3, 4, 8, 15, 17, 18, 41, y 42, presentaron un color castaño claro; dichas líneas pertenecen a la subsp. hypogaea var. hypogaea, mientras que el resto de las líneas presentaron un color rojo oscuro, característico de la subsp. fastigiata var. fastigiata (Fernández y Giayetto 2006).

\section{Porcentaje de frutos maduros e índice de cosecha} En la Tabla 5 se presenta el porcentaje de frutos

Tabla 5. Porcentaje de frutos maduros e índice de cosecha (\% IC) de 22 líneas de maní. Table 5. Percentage of mature fruits and harvest index (\% HI) of 22 peanut lines.

\begin{tabular}{|c|c|c|c|}
\hline \multirow{2}{*}{ Línea } & \multicolumn{2}{|c|}{ Frutos maduros (\%) } & \multirow[t]{2}{*}{$\%$ IC } \\
\hline & Base № & Base peso & \\
\hline 1 & 71,4 bcd & $84,3^{\text {bcde }}$ & $20,4^{\mathrm{abc}}$ \\
\hline 3 & 65,9 bcd & 81,7 bcde & $35,3^{d}$ \\
\hline 4 & $47,9 \mathrm{ab}$ & $68,8^{\text {abcd }}$ & 21,3 abcd \\
\hline 5 & $72,8^{\mathrm{bcd}}$ & 89,0 cde & $22,2^{\text {abcd }}$ \\
\hline 7 & $72,4^{\mathrm{bcd}}$ & 87,3 cde & $21,6^{\text {abcd }}$ \\
\hline 8 & $61,1^{\mathrm{bcd}}$ & $77,1^{\text {bcde }}$ & $28,2^{\mathrm{bcd}}$ \\
\hline 10 & $73,8^{\mathrm{bcd}}$ & 87,2 cde & $23,2^{a b c d}$ \\
\hline 11 & $78,9^{\mathrm{d}}$ & 92,4 de & 25,0 abcd \\
\hline 12 & 48,9 abc & 91,9 de & 25,0 abcd \\
\hline 13 & $81,1^{\mathrm{d}}$ & $62,5 \mathrm{abc}$ & $15,5^{\mathrm{ab}}$ \\
\hline 14 & $78,8^{d}$ & 94,0 de & 24,7 abcd \\
\hline 15 & $81,9^{\mathrm{d}}$ & $88,0^{\text {cde }}$ & 23,3 abcd \\
\hline 16 & $84,4^{\mathrm{d}}$ & 93,0 de & $23,7 \mathrm{abcd}$ \\
\hline 17 & $47,3 \mathrm{ab}$ & $60,1 \mathrm{ab}$ & 16,9 ab \\
\hline 18 & $77,9 \mathrm{~cd}$ & 90,6 de & 34,0 cd \\
\hline 19 & $74,5^{\mathrm{bcd}}$ & $91,1^{\text {de }}$ & $23,2^{a b c d}$ \\
\hline 20 & $88,6^{\mathrm{d}}$ & $97,0^{\mathrm{e}}$ & 23,6 abcd \\
\hline 21 & $60,1^{\mathrm{bcd}}$ & 84,7 bcde & $15,1^{a b}$ \\
\hline 22 & $75,6^{\mathrm{bcd}}$ & $88,0^{\text {cde }}$ & $21,8^{\text {abcd }}$ \\
\hline 23 & $85,6^{\mathrm{d}}$ & $90,7 \mathrm{de}$ & 26,0 bcd \\
\hline 41 & $30,2^{a}$ & $45,1^{\mathrm{a}}$ & $11,2^{a}$ \\
\hline 42 & 63,0 bcd & 85,4 bcde & $23,4^{a b c d}$ \\
\hline C.V.,\% & 21,9 & 16,5 & 30,6 \\
\hline
\end{tabular}

Valores presentados son promedio de tres repeticiones. Letras distintas en la misma columna indican diferencias significativas (Test de Duncan, $\mathrm{P} \leq 0,05$ ).

Previo a su análisis estadístico, los datos fueron transformados utilizando la función $\ln (x+1)$. C.V.: Coeficiente de variación. 
cosechados en base número y peso, e índice de cosecha de las líneas de maní evaluadas, transcurridos 150 días después de siembra (968 DGA). El porcentaje de frutos maduros en base a número y peso varió de 30 a $88 \%$ y de 45 a $97 \%$, respectivamente, mientras que el índice de cosecha varió en un rango de 11 a $35 \%$. El porcentaje de frutos maduros obtenido en base a número es menor al obtenido en base a peso debido a la diferencia de peso de los frutos maduros con respecto a los inmaduros, teniendo este una mayor incidencia en el porcentaje final. Los resultados presentados por esta investigación son similares a lo expuesto por Zapata et al. (2012) quienes obtuvieron porcentajes de frutos maduros en base a número, peso e índice de cosecha a los 148 días después de siembra de 25 a $81 \%$, 41 a $89 \%$, y 6 a 45\%, respectivamente. Fernández y Giayetto (2006) establecen como indicador, para determinar el momento de cosecha del cultivo del maní, la proporción de frutos maduros base número y el índice de cosecha, cuyos parámetros pueden variar ampliamente, considerándose $60 \%$ y $35 \%$ valores aceptables, respectivamente (Kiniry et al., 2005; Fernández y Giayetto, 2006). Al observar estos parámetros se puede apreciar que casi todas las líneas de maní alcanzan la primera condición, exceptuando las líneas 4, 12, 17 y 41, que poseen una proporción de frutos maduros base número menor a $60 \%$. El valor del índice de cosecha obtenido por las distintas líneas en esta investigación fue menor a lo anteriormente mencionado, sin embargo, sólo la línea 3 presentó un índice de cosecha igual a $35 \%$.

En general, la mayor parte de las líneas de maní evaluadas en el presente estudio son de periodo vegetativo más corto que los ecotipos cultivados en Chile, los cuales requieren de 160 a 180 días para que el $80 \%$ de sus frutos alcance madurez de cosecha (Zapata et al., 2012). En este mismo sentido, cabe señalar que la acumulación de grados días en la presente investigación es también menor (968 DGA) al tiempo térmico requerido por diversos genotipos de maní cultivados en Argentina (1500 DGA) (Giayetto et al., 2012).

\section{CONCLUSIONES}

Las líneas 3, 4, 8, 15, 17, 18, 41 y 42 pertenecen a la subsp. hypogaea var. hypogaea; comercialmente las líneas 3 y 8 corresponden al maní tipo Virginia y las restantes líneas corresponden al tipo Runner. Las líneas 1, 5, 7, 10, 11, 12, 13, 14, 16, 19, 20, 21, 22 y 23 corresponden a la subsp. fastigiata var. fastigiata (tipo Valencia).

Las líneas pertenecientes a la subsp. hypogaea presentaron hábito de crecimiento decumbente, patrón de ramificación alternado, color de la semilla castaño claro y frutos que contienen dos semillas. Las líneas de la subespecie fastigiata presentaron hábito de crecimiento erecto, patrón de ramificación secuencial, color de la semilla rojo oscuro y frutos que contienen tres semillas. Las líneas 3 (tipo Virginia), 18 (tipo Runner) y 20 (tipo Valencia), presentaron alto porcentaje de frutos maduros (base a número y peso) e índice de cosecha a los 150 días después de siembra.

\section{RECONOCIMIENTOS}

Este trabajo fue financiado por la Vicerrectoría de Investigación de la Universidad de Concepción, proyecto DIUC N² 211.122.022-1.0.

\section{LITERATURA CITADA}

Del Pozo, A., y P. del Canto. 1999. Áreas agroclimáticas y sistemas productivos en la Vll y Vlll regiones. Serie Quilamapu N 113. INIA Quilamapu, Chillán, Chile.

Di Rienzo J.A., F. Casanoves, M.G. Balzarini, L. Gonzalez, M. Tablada, C.W. Robledo. 2013. InfoStat versión 2013. Grupo InfoStat, FCA, Universidad Nacional de Córdoba, Argentina. URL http://www.infostat.com.ar

Escobar, C., y O. Bustamante. 1997. Estabilidad del rendimiento en semilla de once genotipos de maní (Arachis hypogaea L.) en Santafe de Antioquia. Rev. Fac. Nal. Agron. Medellín 50:55-66.

Ferguson, M.E., P.J. Bramel, and S. Chandra. 2004. Gene diversity among botanical varieties in peanut (Arachis hypogaea L.). Crop Sci. 44:1847-1854.

Fernández, E.M., y O. Giayetto. 2006. El cultivo del maní en Córdoba. Universidad Nacional de Río Cuarto, Río Cuarto, Argentina.

Franco, T.L., y R. Hidalgo. 2003. Análisis estadístico de datos de caracterización morfológica de recursos fitogenéticos. Boletín Técnico IPGRI № 8. Instituto Internacional de Recursos Fitogenéticos, Cali, Colombia.

Giayetto, O., E. Fernández, G. Cerioni, F. Morla, M. Rosso, M. Kearney, y M. Violante. 2012. Caracterización ecofisiológica de genotipos de maní (Arachis hypogaea L.) en Córdoba, Argentina. Ciencia y Tecnología de los Cultivos Industriales 1:201-212.

Harch, B.D., K.E. Basford, I.H. DeLacy, and P.K. Lawrence. 1999. The analysis of large scale data taken from the world groundnut (Arachis hypogaea L.) germplasm collection. II. Two-way data with mixed data types. Euphytica 105:73-82. 
Holbrook, C.C., and H.T. Stalker. 2003. Peanut breeding and genetic resources. Plant Breed. Rev. 22:297-356.

IBPGR. 1985. Groundnut descriptors (revised). International Board for Plant Genetic Resources. International Crop Research Institute for the Semi-Arid Tropics, Rome, Italy.

Kiniry, J.R., C.E. Simpson, A.M. Schubert, and J.D. Reed. 2005. Peanut leaf area index, light interception, radiation use efficiency, and harvest index at three sites in Texas. Field Crops Res. 91:297-306.

Krapovickas, A., and W.C. Gregory. 1994. Taxonomy of the genus Arachis (Leguminosae). Bonplandia (Corrientes) 15:9-206.

Krapovickas, A., R.O. Vanni, J.R. Pietrarelli, D.E. Williams, and C.E. Simpson. 2009. Las razas de maní de Bolivia. Bonplandia (Corrientes) 18:95-189.

Mackliff, J.J., y R.M. Vega. 2013. Evaluación del comportamiento agronómico de 15 líneas de maní del grupo Valencia (Arachis hypogaea L.) en el cantón Caluma, Provincia Bolívar. Tesis de grado Ingeniero Agrónomo. Universidad Estatal de Bolívar, Fac. Ciencias Agropec. Rec. Nat. y Amb., Esc. Ing. Agron, Guaranda, Ecuador.

Mazzani, E., V. Segovia, C. Marín, y W. Pacheco. 2009. Clasificación de cultivares de maní (Arachis hypogaea L.) por caracteres cuantitativos para el establecimiento de colecciones nucleares del banco de germoplasma del Centro Nacional de Investigaciones Agropecuarias, Venezuela. Rev. Científica UDO Agrícola 9:756-763.

Méndez-Natera, y J.R. Osorio. 2003. Evaluación de cultivares de maní (Arachis hypogaea L.) sin la aplicación de fungicidas en épocas de lluvias. Rev. Científica UDO Agrícola 3:47-58.

Pascual, G., S. Molina, C. Morales, K. Valdivia, y F. Quispe. 2006. Extracción y caracterización de aceite de diez entradas de maní (Arachis hypogaea L.) y elaboración de maní bañado con chocolate. Mosaico Científico 3:27-33.
Sánchez-Domínguez, S., A. Muñoz-Orozco, V.A. González-Hernández, y A. MartínezGarza. 2006. Caracterización y clasificación de germoplasma mexicano de cacahuate (Arachis hypogaea L.). Agrociencia 40:171-182.

Satorre, E.H., R.L. Benech, G.A. Slafer, E.B. de la Fuente, D.J. Miralles, M.E. Otegui, y R. Savin. 2003. Producción de granos: bases funcionales para su manejo. Universidad de Buenos Aires, Facultad de Agronomía, Buenos Aires, Argentina.

Soave, J.H., C.A. Bianco, y T.A. Kraus. 2004. Descripción de dos nuevos cultivares de maní (Arachis hypogaea L. subsp. hypogaea var. hypogaea). Agriscientia 21:85-88.

Soave, J., C. Oddino, C. Blanco, S. Soave, A. Moresi, D. Torre, P. Faustinelli, y M. Buteler. 2013. Guasu (AO): primer cultivar argentino de maní tipo Virginia alto oleico. En XXVIII Jornada Nacional del Maní. 19 de septiembre, 2013. CIA / INTA, General Cabrera, Argentina.

Stolpe, N.B. 2006. Descripciones de los principales suelos de la Vlll Región de Chile. Publicaciones del Departamento de Suelos y Recursos Naturales $\mathrm{N}^{\circ} 1$. Universidad de Concepción, Chillán, Chile.

Upadhyaya, H.D., P.J. Bramel, R. Ortiz, and S. Singh. 2002. Developing a mini core of peanut for utilization of genetic resources. Crop Sci. 42:2150-2156.

Upadhyaya, H.D., L.J. Reddy, C.L.L. Gowda, and S. Singh. 2006. Identification of diverse groundnut germplasm: sources of early maturity in a core collection. Field Crops Res. 97:261-271.

Zapata, N., M. Vargas, V.L. Finot, y B. Vallejos. 2012. Caracterización fenológica y morfológica de veinte accesiones de maní (Arachis hypogaea L.) establecidas en la Provincia de Nuble, Chile. Chilean J. Agric. Anim. Sci. (ex AgroCiencia) 28:127-137. 